\title{
X-ray variability of the Narrow Line Seyfert 1 Galaxy PKS 0558-504
}

\author{
W. Brinkmann ${ }^{1}$, P. Arévalo ${ }^{2}$, M. Gliozzi ${ }^{3}$, and E. Ferrero ${ }^{2}$ \\ 1 Centre for Interdisciplinary Plasma Science, Max-Planck-Institut für extraterrestrische Physik, Postfach 1603, \\ 85740 Garching, Germany \\ 2 Max-Planck-Institut für extraterrestrische Physik, Postfach 1603, 85740 Garching, Germany \\ 3 George Mason University, Department of Physics and Astronomy, MS 3F3, 4400 University Dr., Fairfax, VA 22030-4444, \\ USA
}

Received 21 July 2003 / Accepted 20 November 2003

\begin{abstract}
We present results from several XMM-Newton observations of the radio-loud Narrow-line Seyfert 1 galaxy (NLS1) PKS 0558-504. We find evidence for strong and persistent X-ray variability, both on short and long time-scales. On short time scales of $\gtrsim 2$ hours the source varies smoothly by $15-20 \%$; long-term variations by a factor $\gtrsim 2$ could not be resolved in the relatively short exposures: we find the source mostly in a "low" state, in 2 out of the 11 observations in a "high state". X-ray flares seem to be recurrent with a time scale of $\sim 24 \mathrm{ks}$ which, if interpreted as the Keplerian time scale in the disc, would place the emission region just outside the last stable orbit. The X-ray spectrum of PKS 0558-504 can be well fitted by two Comptonization components, one at moderate temperatures of $k T \sim 4.5 \mathrm{keV}$ and optical depths of $\tau \sim 2$, the other at high temperatures $(k T \gtrsim 50 \mathrm{keV})$ and low optical depths $(\tau \lesssim 1.0)$. These parameters are, however, subject to large errors due to the inherent degeneracy of the Comptonization models. Flux variations of the source are caused by changes of the colder component only, the hot component with parameters very similar to those of BLS1 galaxies, stays constant. All results fit nicely the picture that NLS1 galaxies are lower mass objects, accreting close to the Eddington rate emitting X-rays from a very active magnetically powered accretion disc corona.
\end{abstract}

Key words. galaxies: active - galaxies: ISM - galaxies: nuclei - X-rays: galaxies

\section{Introduction}

Narrow-line Seyfert 1 galaxies are optically identified by their emission line properties: the ratio $[\mathrm{O} \mathrm{III}] / \mathrm{H} \beta$ is less than 3 and the $F W H M \mathrm{H} \beta$ less than $2000 \mathrm{~km} \mathrm{~s}^{-1}$ (Osterbrock \& Pogge 1985; Goodrich 1989). Their spectra are further characterized by the presence of strong permitted Fe II, Ca II, and O I $\lambda 8446$ ionization lines (Persson 1988). NLS1 are seldom radio loud (Ulvestad et al. 1995; Siebert et al. 1999; Grupe et al. 1999) and they are usually strong infrared emitters (Moran et al. 1996). In particular, NLS1 have been found to have extreme spectral and variability properties in soft X-rays (Boller et al. 1996; Brandt \& Boller 1998; Boller et al. 2000), which might be related to an extreme value of a primary physical parameter, originating from the vicinity of a super-massive black hole.

PKS 0558-504 $\left(z=0.137, m_{\mathrm{B}}=14.97\right)$ is one of the very few radio-loud NLS1 galaxies $\left(R_{\mathrm{L}}=f_{5 \mathrm{GHz}} / f_{\mathrm{B}} \simeq 27\right.$, Siebert et al. 1999). It was optically identified on the basis of $\mathrm{X}$-ray positions from the High Energy Astronomy Observatory (HEAO-1, Remillard et al. 1986). The Ginga observations (Remillard et al. 1991) showed an increase in the X-ray flux by $67 \%$ in $3 \mathrm{~min}$, implying that the apparent luminosity must be enhanced by relativistic beaming. Further X-ray

Send offprint requests to: W. Brinkmann, e-mail: wpb@mpe.mpg.de observations with different satellites have confirmed the peculiar activity (e.g. strong variability, steep spectrum, high luminosity) of this source. Gliozzi et al. (2000) reported long-term variability from different $\mathrm{X}$-ray observations corresponding to luminosities in the $0.1-2.4 \mathrm{keV}$ soft X-ray band between $1.5 \times$ $10^{45} \mathrm{erg} \mathrm{s}^{-1}$ and $5.4 \times 10^{45} \mathrm{erg} \mathrm{s}^{-1}$ (for $H_{0}=50 \mathrm{~km} \mathrm{~s}^{-1} \mathrm{Mpc}^{-1}$, $q_{0}=0.5$ and isotropic emission). The soft X-ray spectrum is rather steep $(\Gamma \sim 3.1)$ and the medium energy power laws are considerably flatter $(\Gamma \sim 2)$. Another peculiar property displayed by PKS 0558-504 is the unusually high X-ray to radio luminosity ratio (Brinkmann et al. 1997).

The six-days-long ROSAT All Sky Survey light curve of September 1990 and a series of pointed HRI observations (April 19-25, 1998) showed count rate variations by a factor of 2 in less than one day (Gliozzi et al. 2000). With the most extreme value of the luminosity variations found during the observations, $\Delta L / \Delta t=1.12 \times 10^{42} \mathrm{erg} \mathrm{s}^{-2}$, the straightforward application for an estimate of the lower limit of the radiative efficiency of $\eta>4.8 \times 10^{-43} \Delta L / \Delta t$ (Fabian 1979) led to $\eta>0.54$, which exceeds even the theoretical maximum for accretion onto a maximally rotating Kerr black hole. However, spectral variations and the extrapolation of steep power law spectra to low energies can lead to uncertain luminosities, as pointed out by Brandt et al. (1999). 
Table 1. XMM-Newton PN/RGS observations of PKS 0558-504.

\begin{tabular}{|c|c|c|c|c|c|c|}
\hline Orbit & $\begin{array}{c}\text { Observing date } \\
\text { (UT) }\end{array}$ & Instrument & Mode & $\begin{array}{c}\text { Filter } \\
\text { sec }\end{array}$ & $\begin{array}{l}\text { Live time } \\
(\mathrm{ks})\end{array}$ & $\begin{array}{c}\text { count rate } \\
\text { count } / \mathrm{s}\end{array}$ \\
\hline 30 & Feb. 07, 2000: 11:16-13:19 & $\mathrm{PN}$ & $\mathrm{FF}$ & medium & 5.93 & 13.3 \\
\hline 32 & Feb. 10, 2000: 23:26-03:23 & $\mathrm{PN}$ & $\mathrm{FF}$ & thin 2 & 11.49 & 18.2 \\
\hline \multirow[t]{3}{*}{33} & Feb. 12, 2000: 23:13-04:41 & $\mathrm{PN}$ & $\mathrm{FF}$ & thin2 & 16.22 & 17.07 \\
\hline & Feb. 13, 2000: 12:33-17:04 & $\mathrm{PN}$ & FF & thin 1 & 13.34 & 12.41 \\
\hline & Feb. 13, 2000: 18:29-22:59 & $\mathrm{PN}$ & $\mathrm{FF}$ & thin 2 & 13.30 & 13.74 \\
\hline 42 & Mar. 02, 2000: 18:15-21:42 & PN & $\mathrm{FF}$ & medium & 10.93 & 26.0 \\
\hline 45 & Mar. 07, 2000: 20:49-03:31 & $\mathrm{PN}$ & SW & thin1 & 16.88 & 26.9 \\
\hline \multirow[t]{4}{*}{84} & May 24, 2000: 13:04-16:54 & $\mathrm{PN}$ & $\mathrm{FF}$ & medium & 10.68 & 14.5 \\
\hline & $17: 21-21: 08$ & PN & SW & medium & 9.53 & 19.7 \\
\hline & $06: 19-13: 16$ & RGS & SP & - & 25.00 & $\mathrm{n} / \mathrm{a}$ \\
\hline & $13: 16-22: 13$ & RGS & SP & - & 32.21 & $\mathrm{n} / \mathrm{a}$ \\
\hline \multirow[t]{3}{*}{153} & Oct. 10, 2000: 01:49-04:47 & $\mathrm{PN}$ & SW & thin 1 & 7.50 & 18.8 \\
\hline & $05: 38-08: 01$ & PN & FF & thin 1 & 8.05 & 15.3 \\
\hline & $01: 27-08: 46$ & RGS & SP & - & 26.41 & $\mathrm{n} / \mathrm{a}$ \\
\hline \multirow[t]{2}{*}{283} & Jun. 26, 2001: 03:21-06:36 & PN & FF & medium & 10.59 & 12.2 \\
\hline & $02: 37-06: 43$ & RGS & SP & - & 14.80 & $\mathrm{n} / \mathrm{a}$ \\
\hline \multirow[t]{2}{*}{341} & Oct. $19,2001: 12: 41-15: 51$ & $\mathrm{PN}$ & FF & medium & 10.22 & 23.9 \\
\hline & $11: 55-15: 59$ & RGS & SP & - & 14.61 & $\mathrm{n} / \mathrm{a}$ \\
\hline
\end{tabular}

Very dramatic flux variations by a factor of two in $33 \mathrm{~min}$ and, perhaps, by $40 \%$ on a time scale as short as 2 min were reported by Wang et al. (2001) from ASCA observations on January 31, 2000 which leads to an even higher value of the radiative efficiency of $\eta=0.9 \pm 0.2$.

PKS 0558-504 was observed repeatedly by XMM-Newton as a calibration and performance variation (PV) target. The XMM observations benefit from the high sensitivity of the instruments and the uninterrupted exposures resulting from the eccentric satellite orbit. O'Brien et al. (2001) published the spectral analysis of some preliminary data of the commissioning/CalPV phase. The $0.2-10 \mathrm{keV}$ spectrum is dominated by a large soft X-ray excess, which shows no evidence for absorption or emission line features. A power law spectrum at higher energies ( $E \geq 4 \mathrm{keV}$ ) requires in the soft band additionally three black body components; the most physical explanation for the hot big blue bump is, however, Comptonization by a multiple temperature corona of an accretion disc.

Gliozzi et al. (2001) used data from the same observations for a study of the X-ray variability of the source. The long term light curve shows persistent variability with a tendency of the X-ray spectrum to harden when the count rate increases. The short term variability is characterized by smooth modulations with a typical time scale of $\sim 2.2 \mathrm{~h}$ and the most extreme count rate variations found imply a radiative efficiency slightly higher than the theoretical maximum for accretion onto a Schwarzschild black hole.

In this paper we present an analysis of XMM PN and RGS data taken so far in several observations between January 2000 and October 2001. The next section starts with the observational details and a temporal analysis of the data. Section 3 deals with the spectral variability of PKS 0558-504. In Sect. 4 we discuss the results in the framework of disc-corona accretion models for Seyfert galaxies and the last section contains a summary of the main conclusions.

\section{Observations and temporal analysis}

PKS 0558-504 was observed with the instruments on board XMM-Newton during 9 orbits between January 2000 and October 2001. As the source was a calibration target the exposures of the EPIC instruments were always relatively short and different instrument modes were used. For the following analyses we will rely only on data from the RGS instrument (den Herder et al. 2001) and from the PN camera (Strüder et al. 2001). Table 1 lists the relevant data of the observations. As count rate we quote the total number of counts on the chip, divided by the live time. The RGS were always operated in spectroscopy mode (SP), the PN camera in Small Window (SW) or Full Frame (FF) mode, with either a thin or medium filter (for details of the XMM instruments see Ehle et al. 2001).

All PN data have been reprocessed using XMMSAS version 5.4. We selected photons with Pattern $\leq 4$ (i.e. singles and doubles) and quality flag $=0$. We calculated the $0.2-10 \mathrm{keV}$ light curves for all observations by extracting the photons from a circular region centered on the source with a radius of $50^{\prime \prime}$. This extraction radius contains about $90 \%$ of the source photons, using the encircled energy function given by Ghizzardi $\&$ Molendi (2001). We did not correct the light curves for the photon pile up which occurred in the central pixels of the point spread function when the detector was operated in FF mode and the source was in a bright state. It amounts to about $\sim 3 \%$ and does not change the form of the light curve significantly. The backgrounds were determined with the same selection criteria from source free regions on the same chip and subtracted from the source light curves, which were binned in $400 \mathrm{~s}$ bins. 
For four of the orbits the reflection gratings (RGS) provided scientifically useful data simultaneously to the PN exposures. The RGS data was processed using the task rgsproc of the XMMSAS version 5.4. The light curves were constructed using the task evselect, selecting photons in the $0.3-2.0 \mathrm{keV}$ energy band that belonged both to the source spatial region and the first order spectral region. The background light curves were calculated from all photons belonging to the first order spectral region and to the spatial region excluding the source. As the detector area used for the background is larger than the area of the source the background light curve had to be rescaled before being subtracted. The background subtracted source light curve was binned in $1000 \mathrm{~s}$ bins to achieve a reasonable signal to noise ratio while preserving the overall shape of the curve.

The PN data is affected by the dead time of the detector that reduces the effective exposure by a factor of 0.9994 in the FF mode and 0.7101 in the SW mode. There is also a loss of counts due to the out-of-time events, the photons collected during the read out. This last effect further reduces the collection efficiency by a factor of 0.9328 in the FF and 0.9884 in the SW mode, respectively. The PN light curves were thus corrected for these two effects. The RGS light curves were scaled by a factor of $\gtrsim 12$ in order to match them with the resulting PN SW count rates of the corresponding orbits. The plots in Fig. 1 show the PN light curves of early orbits for which no RGS observations were performed. In Fig. 2 we present the later PN observations with the scaled RGS light curves overlaid so that the times match. We used the same length for most of the time axes and the same range of count rates for a comparison of the variability pattern. Only orbit 33 and 84 had considerably longer exposures.

Clearly visible is a deficit of counts in the FF mode of $\sim 20 \%$ when the RGS light curve is normalized to the SW mode data. This is an effect of photon pile up and a redistribution of double events into higher order event patterns (Freyberg, private comm). The correction factor to the RGS data is in this case of pattern migration of the order of $\gtrsim 15$, but it is, as for the SW mode data, not the same for all orbits. It obviously does not only depend on the count rate and spectral variations but seems to be influenced by the level of background activity as well. For the following correlations of source properties with source intensities we will use normalized count rates, obtained by detailed corrections for the above instrumental effects.

On long time scales (different orbits) the light curves show that the source undergoes intensity variations by a factor of $\gtrsim 2$. During the individual exposures we find substantial variability with an amplitude of up to $\sim 15-20 \%$, which is not correlated with the average count rate of the observation. We never detected the large intensity variations in an individual exposure, thus the time scale must be longer than typically one orbit. Figure 4 (see Sect. 2.1) further suggests that the source is mainly found in a low state, with only relatively rare high states. The variances in the hard and soft band light curves are very similar; only in the highest intensity states the hard band light curves show stronger variations than the soft light curves.

The relatively smooth and nearly linear intensity changes have a time scale of typically $\gtrsim 2 \mathrm{~h}$ as can be seen directly from the light curves (Figs. 1 and 2). However, this is not a strict,
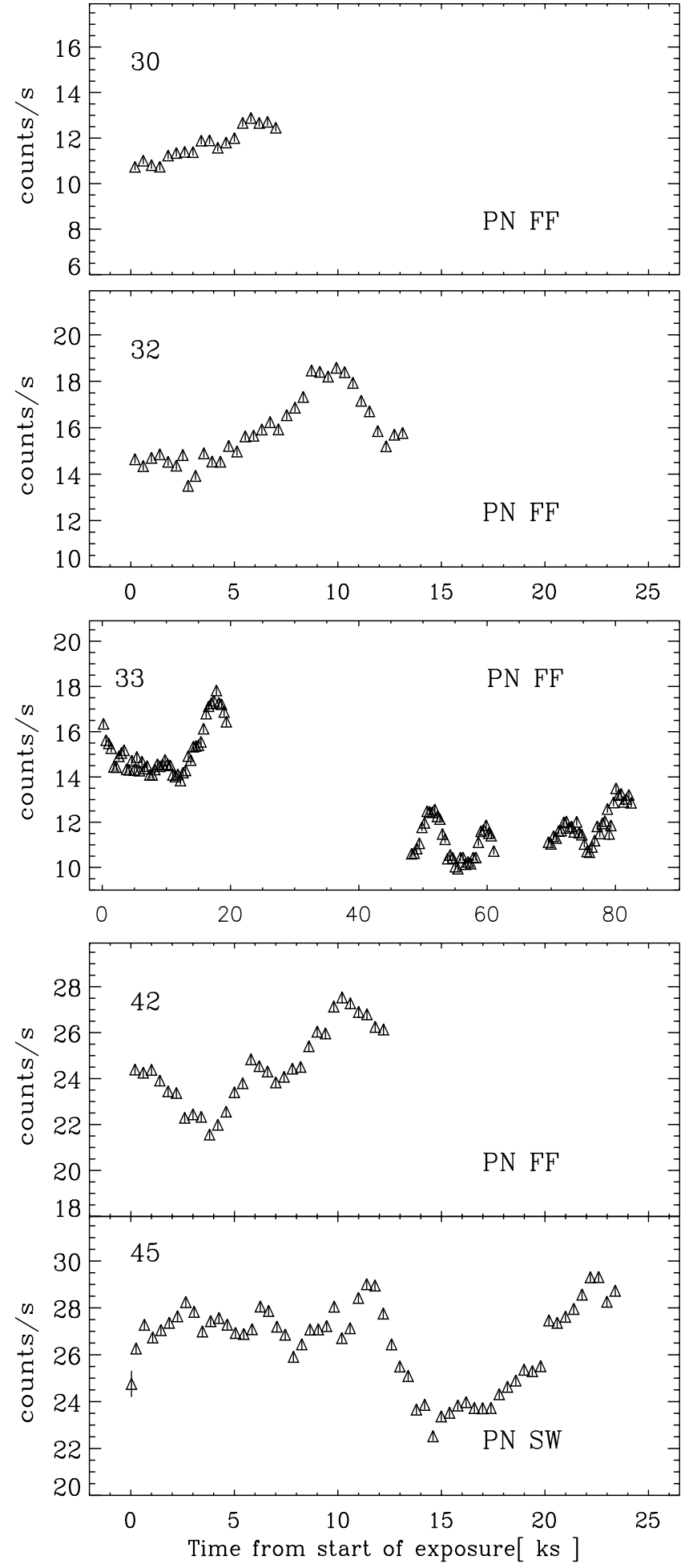

Fig. 1. Light curves of PKS 0558-504 for the early orbits without RGS exposures. Triangles represent the PN data, with error bars smaller than the symbols. Note the longer time axis for orbit 33.

well defined time scale as illustrated by the structure functions (Simonetti et al. 1985) of some individual orbits (Fig. 3). As already noted by Gliozzi et al. (2001) this time scale merely seems to reflect the typical rise/decay time scales in the light curves with a relatively narrow variance. But the light curves 

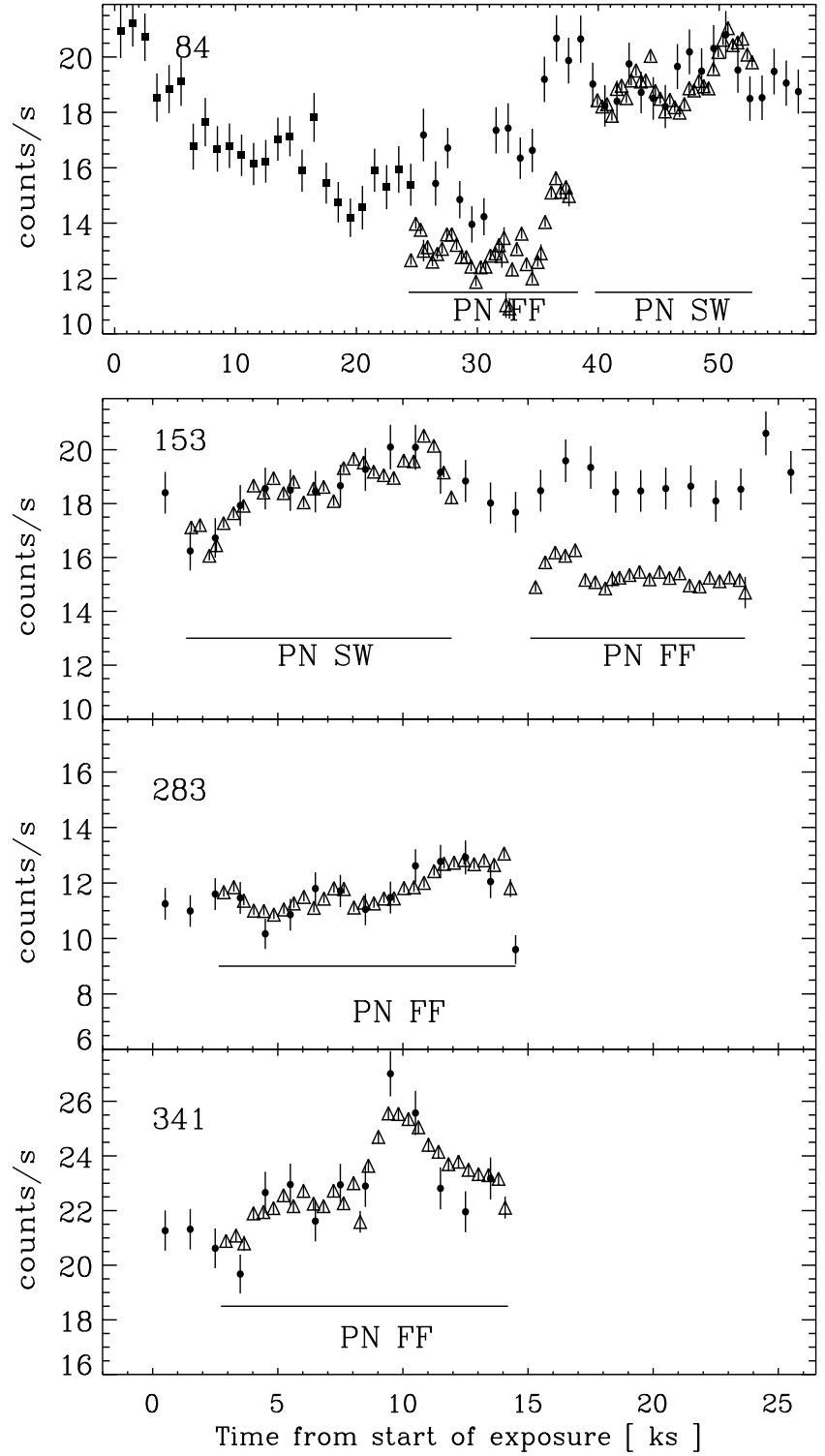

Fig. 2. Light curves of PKS 0558-504 for the later orbits with RGS exposures. Triangles represent the PN data, with error bars smaller than the symbols. The dots represent the RGS light curves rescaled to match the PN SW count rate of the corresponding exposure. For orbit 84 the filled squares represent the first RGS exposure using both RGS detectors while for the second RGS exposure (dots) only the RGS 2 was available.

show as well that even the longest RGS exposure in orbit 84 is too short to cover all relevant time scales of the source.

The temporal structure of the intensity variations during different orbits show some intriguing similarities in shape and even in the amplitudes of the flux variations, independent of the average flux level. A temporal analysis of the whole data set, using orbit 32 as a template, shows a "repetition rate" of that signal of $\sim 23850 \mathrm{~s}$. The search was simply done by aligning the count rate peak of orbit 32 with the individual peaks of the other light curves. If the signal were strictly periodic we would expect this flare in 8 of the orbits but we find only 6 at the expected position. There are two exposures, orbits 153 and 283 , when the source is in a low state, where no flare is

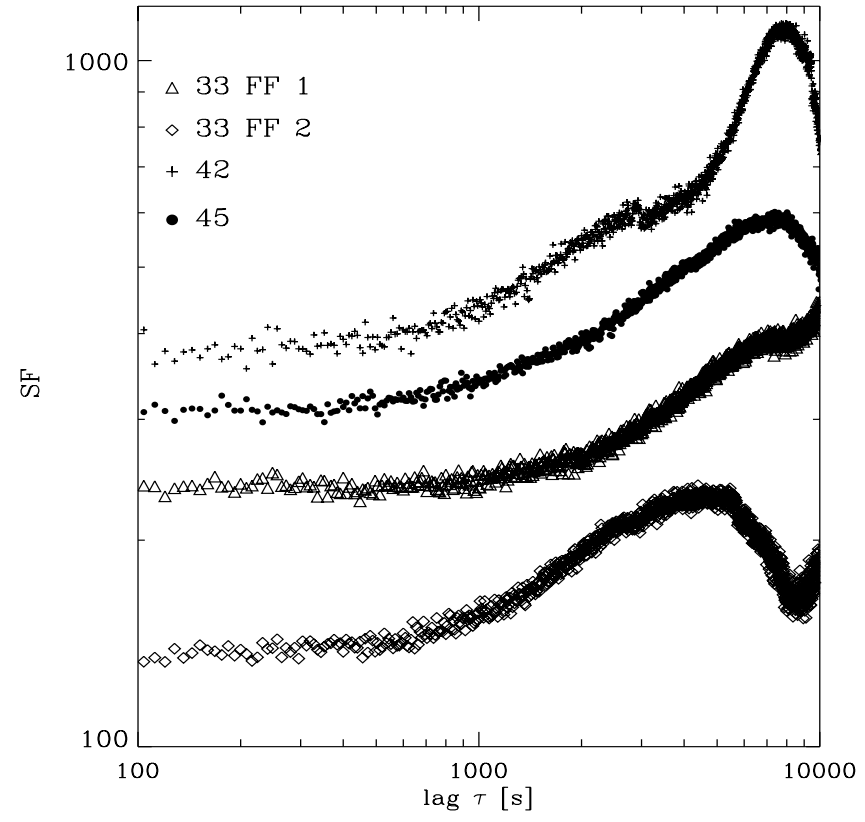

Fig. 3. Structure functions of the light curves of several orbits. The graphs are displaced arbitrarily in $y$-direction for clarity.

found at the expected time. The statistical significance for this "quasi-periodic" repetition would be rather high for purely random fluctuations, but lower for other models like shot-noise or self-organized criticality in the accretion disc (Mineshige et al. 1994). A rigorous statistical analysis to determine the significance of the existence of that signal, like the Monte Carlo technique developed by Uttley et al. (2002) turned out to be impossible, due to the underlying red noise process and, in particular, due to the extreme sparsity of the data, covering observations over more than two years. Longer observations are clearly required for definite results.

The most dramatic flux variations occurs near the middle of orbit 341 where the count rate changes by $\sim 4.3$ counts $\mathrm{s}^{-1}$ in $\sim 1128 \mathrm{~s}$. With the spectral parameters deduced below, assuming a Friedman cosmology with $H_{\mathrm{o}}=75 \mathrm{~km} \mathrm{~s}^{-1} \mathrm{Mpc}^{-1}$ and $q_{\mathrm{o}}=0.5$, we obtain a $\Delta L_{0.2-10 \mathrm{keV}} / \Delta t \sim 4.5 \times 10^{41} \mathrm{erg} \mathrm{s}^{-2}$. This leads to an upper limit of the radiative efficiency (Fabian 1979) of $\eta \sim 0.23$ which is similar to that found previously from ROSAT (Gliozzi et al. 2000) and XMM observations (O'Brien et al. 2001). These efficiency values are much more moderate than those claimed for the two flares detected by Ginga (Remillard et al. 1991) and Wang et al. (2001).

\subsection{Hardness ratios}

We computed hardness ratios using $1000 \mathrm{~s}$ binned soft and hard band light curves, and the expression, $H R=$ [4.0-10 keV]/[0.2-1.0 keV]. The count rates in these energy bands appear to represent the two relatively independent spectral components of the source, as can be seen in Sect. 4. By plotting the $H R$ as a function of the total count rate $(0.2-10 \mathrm{keV})$, we are able to investigate whether the $H R$ variations are correlated with the source flux. 


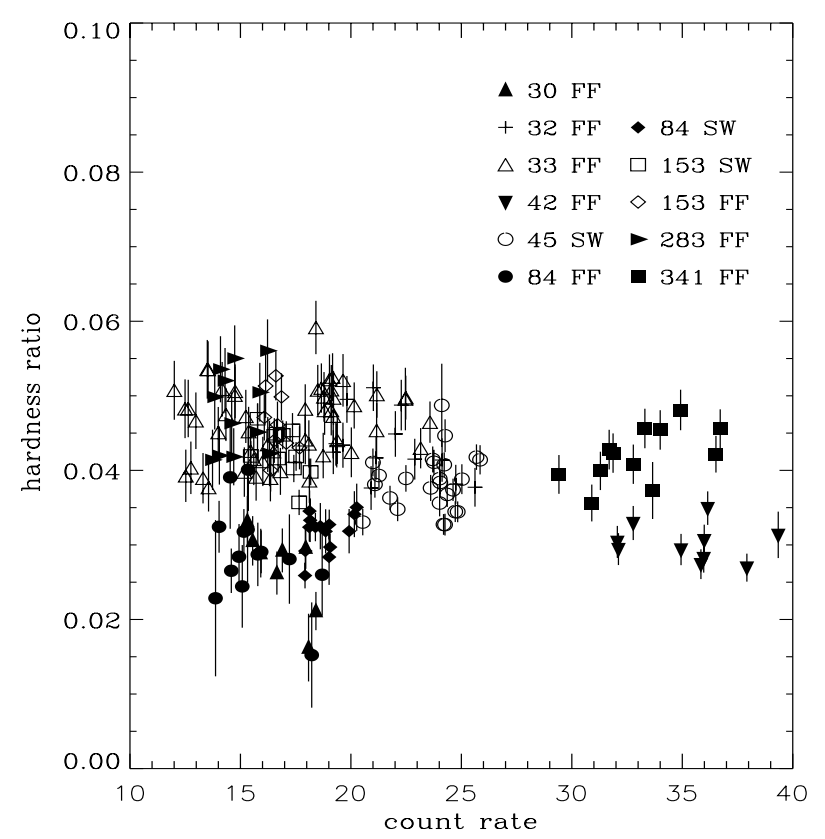

Fig. 4. Hardness ratios as function of the total count rate for different orbits. Filled symbols denote observations with medium filter, open symbols with thin filters.

The total count rates were corrected for the above mentioned dead-time effects as well as for pattern migration and pile up. From spectral simulations with typical source parameters (see Sect. 3) we found that the hardness ratios change by about 0.01 comparing the thin filter observations with the medium filter data.

In Fig. 4 we plot the hardness ratios as function of the corrected $0.2-10 \mathrm{keV}$ count rate for the different orbits. Filled symbols denote observations with medium filter, open symbols with thin filter. The count rates and hardness ratios are corrected to medium filter observations. Some data points with large errors are affected by flares in the background. We do not find a simple correlation between hardness ration and count rate as claimed by Gliozzi et al. (2001) from their restricted data base. The data suggest that the source can be predominantly found in a lower intensity state, differing by a factor of two in count rate from the high states. This reflects the skew distribution of count rates found in other NLS1 as well. Whether the gap between these two states is real or a selection effect due to the limited observational coverage, can only be decided in future observations. The hardness ratios show a large dispersion on long time scales, i.e. for different orbits. On the shorter time scales of a typical observation the hardness ratios in the low state appear to be nearly constant. There is a general slight tendency for the hardness ratios to get smaller with increasing count rate. In the high state orbit 341 there are strong indications of a hardening of the spectrum when the source brightens.

\section{Spectral analysis}

NLS1 galaxies are generally characterized by very steep spectra in the soft energy band (Boller et al. 1996). From ROSAT observations of PKS 0558-504 Gliozzi et al. (2000) find a steep power law with $\Gamma \sim 3$ in the $0.1-2.4 \mathrm{keV}$ energy range;

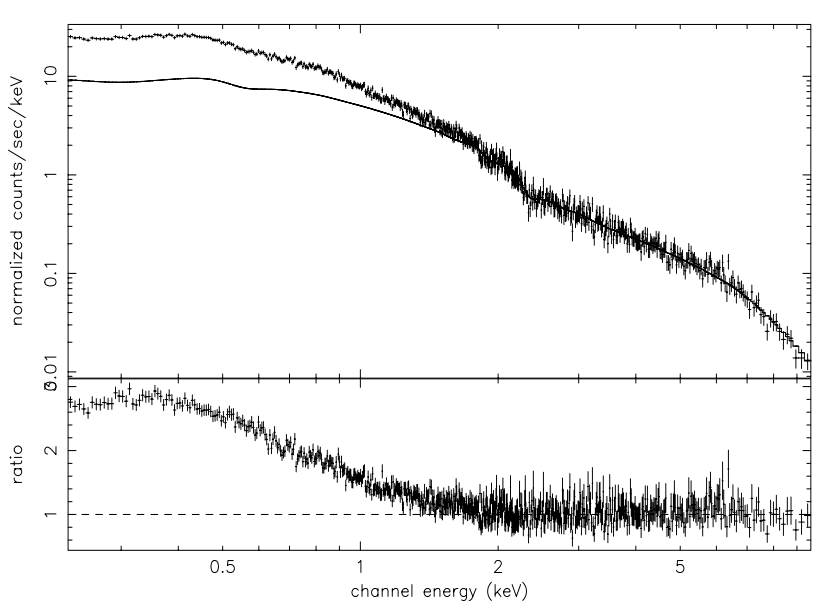

Fig. 5. Power law fit with galactic absorption to the PN data for PKS 0558-504 in the 2-10 keV energy range; the fitted model is extrapolated to lower energies. The lower panel gives the ratio between data and model.

in the ASCA $0.6-10 \mathrm{keV}$ band Vaughan et al. (1999) obtain $\Gamma=2.26 \pm 0.03$. The XMM-Newton data (O'Brien et al. 2001) clearly show a strong soft excess below $\sim 2 \mathrm{keV}$ over a harder power law at higher energies.

The PN data with their outstanding signal to noise ratio and their wide bandpass are ideally suited for a detailed spectral study of the source. For the spectral analysis we used the latest available response matrices (version 6.3) issued in December 2002. We extracted single and double events with quality flag $=0$ from a rectangular region of $30 \times 30$ RAW pixels around the source position. The region includes $\sim 90 \%$ of the source photons but avoids the gap between the detector chips. The background was taken on the same chip at distances as close to the source position as possible, avoiding contamination. With a count rate of $\gtrsim 20$ counts $\mathrm{s}^{-1}$ in the high state the PN detector, operated in Full Window mode, showed strong indications of pile-up, clearly apparent from the XMMSAS task epatplot. We therefore discarded photons from the innermost (typically $2 \times 3$ ) RAW pixels at the core of the point spread function from the spectral analysis.

In Fig. 5 we show the power law fit in the $2-10 \mathrm{keV}$ energy range to the data of orbit $153\left(\Gamma=2.13 \pm 0.03 ; \chi_{\text {red }}^{2}=\right.$ $0.90 / 303$ d.o.f. with a galactic $N_{\mathrm{H}}=4.4 \times 10^{20} \mathrm{~cm}^{-2}$ ). The fitted model is extrapolated to lower energies and the ratio between data and model, given in the lower panel, clearly demonstrates the presence of a large soft X-ray excess over the hard power law. The spectrum of PKS 0558-504 appears to be a carbon copy of that of the NLS1 galaxy PG 0844+349 (Brinkmann et al. 2003) even with respect to the "big blue bump" seen in both objects (O`Brien et al. 2001) and it is very similar to that of 1H 0419-577 (Page et al. 2002) and the other NLS1 galaxy Mrk 896 (Page et al. 2003) studied with XMM-Newton.

The upper limits given for an iron line are rather low (O'Brien et al. 2001) and the soft band spectral excess is far too broad to be fitted by a single black body component. A multiple blackbody (in the soft band) plus a power law at higher energies provides acceptable fits to the data, however, the physical nature of these different components remains obscure. While a 


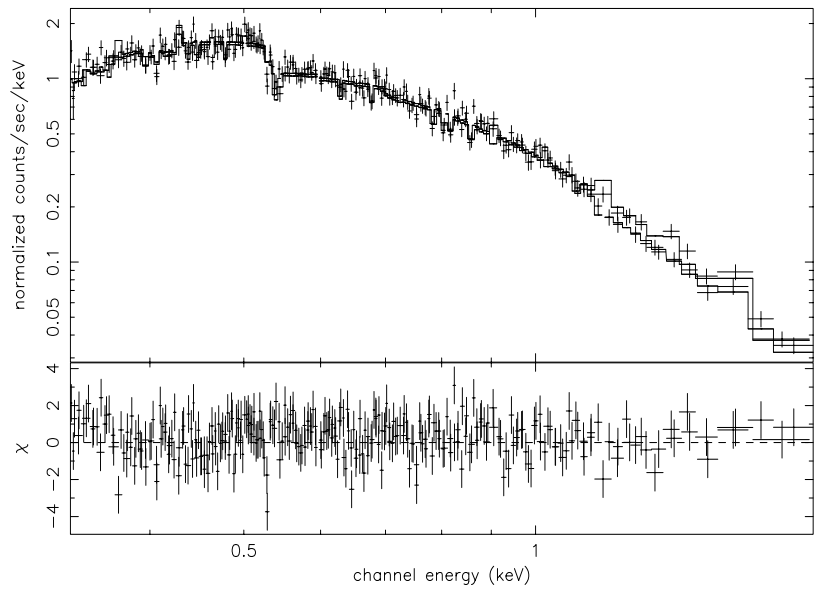

Fig. 6. CompTT + power law fit (without edge) with galactic absorption to the RGS1 data of orbit 84 . The lower panel gives the $\Delta \chi^{2}$ per channel.

model with two Comptonization components gives an accurate description of the spectra, two power law models require absorption in excess of the galactic value and yield slightly worse fits. For example, for the above mentioned orbit 153 (see also Table 2) we obtain an $N_{\mathrm{H}}=7.79 \times 10^{20} \mathrm{~cm}^{-2}, \Gamma_{\text {soft }}=3.28 \pm$ $0.08, \Gamma_{\text {hard }}=1.62 \pm 0.10$ with a $\chi_{\text {red }}^{2}=1.163 / 578$ d.o.f., values, which are representative for the other orbits as well.

The relatively long RGS observation of orbit 84 provides a sufficient number of photons for an accurate fit. We have reprocessed the RGS data using XMMSAS version 5.3.3 and the RGS response matrices were created with the SAS package rgsrmfgen. The spectral data were binned to contain at least 30 photons per energy channel. We fitted the RGS data with a single compTT Comptonization model, available in Xspec (Titarchuk 1994), assuming galactic absorption plus an additional high energy power law (see Sect. 3.1). The fit is acceptable $\left(\chi_{\text {red }}^{2}=1.01 / 1162\right.$ d.o.f. $)$ and yielded a temperature of the soft photons of $k T_{0}=63.9 \pm 5.0 \mathrm{eV}$ and an optical depth of $\tau=2.47 \pm 0.25$. The temperature of the hot Comptonizing electrons was fixed at $k T_{1}=4.5 \mathrm{keV}$ to reduce the error bars (see the discussion below). An additional absorption edge, required only for one of the two observation intervals $\left(E_{\text {edge }}=1.14 \pm 0.02 \mathrm{keV}, \tau=0.27 \pm 0.06\right)$ improves the fit significantly $\left(\chi_{\text {red }}^{2}=0.989 / 1158\right.$ d.o.f.) according to an F-test. The residuals shown in Fig. 6 are, however, smaller than the systematic differences between the two RGS detectors and no other strong absorption or emission features are observed.

We tried to determine the possible contribution of an iron line (neutral or ionized) to the spectra. To improve the signal to noise ratio we accumulated the data from different observation modes and filters and fitted first a power law and then a power law with a line to the combined SW and FF-mode 4-9 keV data. As the accumulated spectra for the different modes covered different intensity states of the source, the normalizations of the spectra were different for the SW and FF mode data. For a neutral iron line we obtained $90 \%$ upper limits for the equivalent widths of $E W<25 \mathrm{eV}$ (SW) and $<35 \mathrm{eV}$ for the FF-mode data; for an ionized line we obtained $E W<54 \mathrm{eV}$ (SW) and $<72 \mathrm{eV}$ for the FF-mode data, using a line width of $\sigma=0.1 \mathrm{keV}$ for the line. For narrow lines $(\sigma=0.01 \mathrm{keV})$ the corresponding limits are nearly a factor of 2 lower. In all fits the inclusion of the lines did not improve the fits. The single power law model yielded already $\chi_{\text {red }}^{2}=0.927$, thus the data do not require the presence of any iron emission line.

The featureless spectrum indicates that we are seeing the bare continuum disk emission from the quasar: thus disk Comptonization models, where the $\mathrm{X}$-rays are produced via inverse Compton emission in a hot corona embedding a cooler accretion disk (e.g. Haardt \& Maraschi 1993; Pounds et al. 1995), provide a satisfactory physical explanation to the data.

In a first step we fitted the combined PN data of each orbit, regardless of flux variations during the observation with the sum of two compTT components with absorption fixed at the galactic value. This Comptonization model describes the upscattering of soft photons by hot, thermal electrons in a corona above the accretion disc. The temperatures of the soft photons were assumed to be the same for both components. In Table 2 we present the results of the fits for the different orbits and observation modes. The temperatures of the soft photons, given in Col. 1 are always around $80 \mathrm{eV}$, with relatively small errors. The temperatures of the Comptonizing electrons of the first component turned out to be $k T_{1}=4.5-5 \mathrm{keV}$, the optical depths around $\tau_{1} \sim 2$, both with rather large errors. The second component is characterized by rather high temperatures, $k T_{2} \gtrsim$ $50 \mathrm{keV}$, but optical depths $\tau_{2} \lesssim 1.0$, and both parameters have very large errors.

The physical parameters in the Comptonization models are only poorly constrained because the spectral shape in the X-ray range is mainly determined by the combination of the temperature $k T / \mathrm{mc}^{2}$ and the optical depth $\tau$ of the scattering electrons; the cutoff energy is essentially related to $k T / \mathrm{mc}^{2}$. For Comptonizing electrons as hot as found from the fits, the determining spectral characteristics (high energy cut-off) is at energies far above the energy range of the XMM instruments and only the spectral index $\alpha$ of the non-relativistic, low-energy part of the spectrum can be obtained (Titarchuk \& Lyubarskij 1995). Variations of the spectral shape with intensity, time lags between the fluxes in different energy bands, or energy resolved power density spectra as additional diagnostic tools (e.g. Kazanas et al. 1997) might provide insight into the way the corona is heated and cooled.

\subsection{High energy power law fits}

While the cooler Comptonization model nicely matches the PN energy band only the power law like, low energy part of the hot Comptonizing component falls into the energy band $\leq 10 \mathrm{keV}$. We therefore fitted the data with a combination of a compTT plus a power law model. The temperature of the Comptonizing photons was fixed at $k T=4.5 \mathrm{keV}$ and the absorption to the galactic value.

Table 3 summarizes the results of these fits. The parameters of the Comptonization model are rather well determined, the errors of the normalization are typically $\$ 6 \%$. The slopes of the high energy power laws are reasonably accurate, the normalizations of the power laws show, however, rather large 
Table 2. Results from spectral fits of two-component Comptonization models assuming fixed Galactic $N_{\mathrm{H}}=4.4 \times 10^{20} \mathrm{~cm}^{-2}, z=0.137$.

\begin{tabular}{|c|c|c|c|c|c|c|c|c|c|}
\hline $\begin{array}{l}\text { Orbit, } \\
\text { mode }\end{array}$ & $\begin{array}{l}k T_{\mathrm{o}} \\
(\mathrm{eV})\end{array}$ & $\begin{array}{c}k T_{1} \\
(\mathrm{keV})\end{array}$ & $\tau_{1}$ & norm $_{1}$ & $\begin{array}{c}k T_{2} \\
(\mathrm{keV})\end{array}$ & $\tau_{2}$ & norm $_{2}$ & $\chi_{\text {red }}^{2}$ & d.o.f \\
\hline $30 \mathrm{FF}$ & $77.1_{-10.2}^{+10.2}$ & $4.5_{-4.5}^{+166.8}$ & $1.9_{-1.9}^{+60.0}$ & $1.30 \mathrm{E}-2$ & $47.3_{-47.3}^{+2614}$ & $0.72_{-0.72}^{+51.77}$ & $1.90 \mathrm{E}-4$ & 1.098 & 464 \\
\hline $32 \mathrm{FF}$ & $87.4_{-5.6}^{+5.6}$ & $4.6_{-4.6}^{+74.8}$ & $2.4_{-2.4}^{+29.7}$ & $1.21 \mathrm{E}-2$ & $61.8_{-61.8}^{+3929}$ & $0.93_{-0.93}^{+70.16}$ & $0.98 \mathrm{E}-4$ & 1.026 & 716 \\
\hline $33 \mathrm{FF}(1)$ & $84.8_{-5.1}^{+5.1}$ & $4.4_{-4.4}^{+68.4}$ & $2.3_{-2.3}^{+27.5}$ & $1.10 \mathrm{E}-2$ & $52.4_{-52.4}^{+1960}$ & $0.77_{-0.77}^{+36.66}$ & $1.97 \mathrm{E}-4$ & 1.039 & 823 \\
\hline $33 \mathrm{FF}(2)$ & $86.9_{-6.9}^{+6.9}$ & $5.3_{-5.3}^{+119.6}$ & $1.9_{-1.9}^{+37.7}$ & $0.71 \mathrm{E}-2$ & $60.2_{-60.2}^{+2869}$ & $0.73_{-0.73}^{+44.73}$ & $0.96 \mathrm{E}-4$ & 1.089 & 639 \\
\hline $33 \mathrm{FF}(3)$ & $82.8_{-7.4}^{+7.4}$ & $5.3_{-5.3}^{+138.3}$ & $1.8_{-1.8}^{+42.0}$ & $0.86 \mathrm{E}-2$ & $61.2_{-61.2}^{+2144}$ & $0.63_{-0.63}^{+29.34}$ & $1.34 \mathrm{E}-4$ & 1.088 & 663 \\
\hline $42 \mathrm{FF}$ & $86.7_{-5.3}^{+5.3}$ & $4.5_{-4.5}^{+68.38}$ & $2.4_{-2.4}^{+27.8}$ & $2.43 \mathrm{E}-2$ & $51.4_{-51.4}^{+2502}$ & $0.60_{-0.60}^{+40.15}$ & $3.38 \mathrm{E}-4$ & 1.029 & 757 \\
\hline $45 \mathrm{SW}$ & $74.7_{-6.2}^{+6.2}$ & $4.8_{-4.8}^{+103.8}$ & $1.9_{-1.9}^{+35.7}$ & $3.37 \mathrm{E}-2$ & $49.9_{-49.9}^{+2282}$ & $1.08_{-1.08}^{+55.46}$ & $2.74 \mathrm{E}-4$ & 1.002 & 909 \\
\hline $84 \mathrm{FF}$ & $78.8_{-17.4}^{+17.4}$ & $4.5_{-4.5}^{+241.3}$ & $1.7_{-1.7}^{+84.0}$ & $1.59 \mathrm{E}-2$ & $49.9_{-49.9}^{+2782}$ & $0.59_{-0.59}^{+45.27}$ & $2.92 \mathrm{E}-4$ & 0.957 & 415 \\
\hline $84 \mathrm{SW}$ & $73.4_{-11.8}^{+11.8}$ & $4.6_{-4.6}^{+187.5}$ & $1.8_{-1.8}^{+65.9}$ & $2.73 \mathrm{E}-2$ & $52.8_{-52.8}^{+2659}$ & $0.72_{-0.72}^{+46.16}$ & $3.05 \mathrm{E}-4$ & 1.029 & 485 \\
\hline $153 \mathrm{FF}$ & $87.3_{-8.8}^{+8.8}$ & $4.5_{-4.5}^{+112.3}$ & $2.0_{-2.0}^{+42.5}$ & $0.98 \mathrm{E}-2$ & $49.8_{-49.8}^{+2440}$ & $0.72_{-0.72}^{+46.25}$ & $1.98 \mathrm{E}-4$ & 1.067 & 576 \\
\hline $153 \mathrm{SW}$ & $77.0_{-7.8}^{+7.8}$ & $4.7_{-4.7}^{+119.7}$ & $1.9_{-1.9}^{+42.1}$ & $2.18 \mathrm{E}-2$ & $50.5_{-50.5}^{+1974}$ & $0.79_{-0.79}^{+38.96}$ & $3.00 \mathrm{E}-4$ & 0.974 & 656 \\
\hline $283 \mathrm{FF}$ & $87.8_{-10.1}^{+10.1}$ & $5.2_{-5.2}^{+184.5}$ & $1.7_{-1.7}^{+55.3}$ & $0.68 \mathrm{E}-2$ & $40.0_{-40.0}^{+1135}$ & $0.83_{-0.83}^{+29.74}$ & $3.97 \mathrm{E}-4$ & 1.119 & 695 \\
\hline $341 \mathrm{FF}$ & $93.1_{-6.5}^{+6.5}$ & $5.0_{-5.0}^{+122.8}$ & $2.1_{-2.1}^{+42.6}$ & $0.80 \mathrm{E}-2$ & $46.3_{-46.3}^{+1073}$ & $0.51_{-0.51}^{+13.86}$ & $6.32 \mathrm{E}-4$ & 1.108 & 792 \\
\hline
\end{tabular}

NOTE: For orbits $<45$ the lower threshold for the event amplitudes was different from the later setting. No extra response matrices exist for these early observations, but the spectral fits seem not to be affected noticeably.

Table 3. Results from spectral fitting of a compTT plus a hard power law model, assuming fixed galactic $N_{\mathrm{H}}=4.4 \times 10^{20} \mathrm{~cm}^{-2}, z=0.137$.

\begin{tabular}{|c|c|c|c|c|c|c|c|c|}
\hline $\begin{array}{l}\text { Orbit, } \\
\text { mode }\end{array}$ & $\begin{array}{l}k T_{\mathrm{o}} \\
(\mathrm{eV})\end{array}$ & $\begin{array}{c}k T \\
(\mathrm{keV})\end{array}$ & $\tau$ & norm $_{\text {compTT }}$ & $\bar{\Gamma}$ & norm $_{\mathrm{po}}$ & $\chi_{\text {red }}^{2}$ & d.o.f. \\
\hline $30 \mathrm{FF}$ & $75.1 \pm 2.8$ & 4.5 & $1.81 \pm 0.14$ & $2.29 \mathrm{E}-02$ & $1.91 \pm 0.12$ & $2.40 \mathrm{E}-03$ & 1.104 & 466 \\
\hline $32 \mathrm{FF}$ & $89.1 \pm 3.1$ & 4.5 & $2.09 \pm 0.19$ & $1.81 \mathrm{E}-02$ & $1.93 \pm 0.14$ & $3.84 \mathrm{E}-03$ & 1.033 & 718 \\
\hline $33 \mathrm{FF}(1)$ & $88.0 \pm 3.0$ & 4.5 & $1.87 \pm 0.19$ & $1.51 \mathrm{E}-02$ & $2.01 \pm 0.08$ & $4.86 \mathrm{E}-03$ & 1.045 & 825 \\
\hline $33 \mathrm{FF}(2)$ & $92.5 \pm 3.3$ & 4.5 & $1.88 \pm 0.20$ & $1.07 \mathrm{E}-02$ & $1.97 \pm 0.08$ & $2.78 \mathrm{E}-03$ & 1.082 & 641 \\
\hline $33 \mathrm{FF}$ (3) & $87.7 \pm 2.7$ & 4.5 & $1.76 \pm 0.17$ & $1.34 \mathrm{E}-02$ & $1.97 \pm 0.09$ & $3.24 \mathrm{E}-03$ & 1.076 & 665 \\
\hline $42 \mathrm{FF}$ & $91.0 \pm 4.4$ & 4.5 & $2.24 \pm 0.23$ & $2.67 \mathrm{E}-02$ & $2.12 \pm 0.17$ & $6.43 \mathrm{E}-03$ & 1.025 & 759 \\
\hline $45 \mathrm{SW}$ & $76.3 \pm 1.2$ & 4.5 & $1.94 \pm 0.06$ & $2.67 \mathrm{E}-02$ & $1.80 \pm 0.07$ & $2.37 \mathrm{E}-03$ & 1.040 & 856 \\
\hline $84 \mathrm{FF}$ & $78.8 \pm 3.4$ & 4.5 & $1.71 \pm 0.19$ & $1.91 \mathrm{E}-02$ & $1.93 \pm 0.14$ & $2.51 \mathrm{E}-03$ & 0.953 & 417 \\
\hline $84 \mathrm{SW}$ & $74.1 \pm 2.6$ & 4.5 & $1.83 \pm 0.13$ & $2.66 \mathrm{E}-02$ & $1.85 \pm 0.12$ & $2.56 \mathrm{E}-03$ & 1.025 & 487 \\
\hline $153 \mathrm{FF}$ & $91.3 \pm 3.5$ & 4.5 & $1.82 \pm 0.22$ & $1.34 \mathrm{E}-02$ & $1.95 \pm 0.11$ & $3.46 \mathrm{E}-03$ & 1.060 & 578 \\
\hline $153 \mathrm{SW}$ & $77.7 \pm 1.8$ & 4.5 & $1.93 \pm 0.09$ & 2.14E-02 & $1.81 \pm 0.08$ & $2.46 \mathrm{E}-03$ & 0.971 & 658 \\
\hline $283 \mathrm{FF}$ & $90.1 \pm 3.5$ & 4.5 & $1.68 \pm 0.22$ & $0.96 \mathrm{E}-02$ & $1.97 \pm 0.07$ & $3.90 \mathrm{E}-03$ & 1.116 & 697 \\
\hline $341 \mathrm{FF}$ & $93.4 \pm 5.4$ & 4.5 & $2.41 \pm 0.29$ & $1.99 \mathrm{E}-02$ & $2.00 \pm 0.17$ & $6.60 \mathrm{E}-03$ & 1.109 & 794 \\
\hline
\end{tabular}

errors which can amount to $\$ 30 \%$ during the high states, when the power law contribution to the total spectrum is small.

In Fig. 7 we show the dependencies of fitted parameters of the Comptonization component on the corresponding count rates of the observation interval. The temperature $T_{0}$ of the soft input photons (bottom panel) remains around $80 \mathrm{eV}$, with no clear correlation with the intensity state of the source (a linear fit yielded a slope of $\left.\beta=(0.290 \pm 0.299) \times 10^{-3}\right)$. The optical depth shows a linear correlation with count rate with a slope of $\beta=(0.272 \pm 0.040) \times 10^{-1}$. The parameters of the power law components of the same fits are shown in Fig. 8. The spectral index remains nearly constant (slope of linear fit $\beta=(0.593 \pm$ $0.324) \times 10^{-2}$ ) at a value of 2.0 . Note, that in most plots the intrinsic variance of the data is quite high.

The normalization of the power law changes only marginally and quite generally, the errors of the fitted parameters are larger when the source is bright and thus the relative contribution of the power law component smaller. Changes of the flux are thus mainly caused by an increase of the optical depth of the scattering electrons combined with an increase of the normalization of the Comptonization component.

We repeated the fits, leaving the temperature of the scattering electrons free but fixed the optical depth to $\tau=1.8$. The results are very similar to those of Fig. 7. The flux variations are in that case correlated linearly only with changes of the temperature of the scattering electrons over the range $3.9 \mathrm{keV} \lesssim k T \lesssim$ $7.4 \mathrm{keV}$.

\section{Discussion}

\subsection{Spectral properties}

The spectrum of PKS 0558-504, like that of other NLS1 galaxies, can be fitted well by a combination of two compTT 


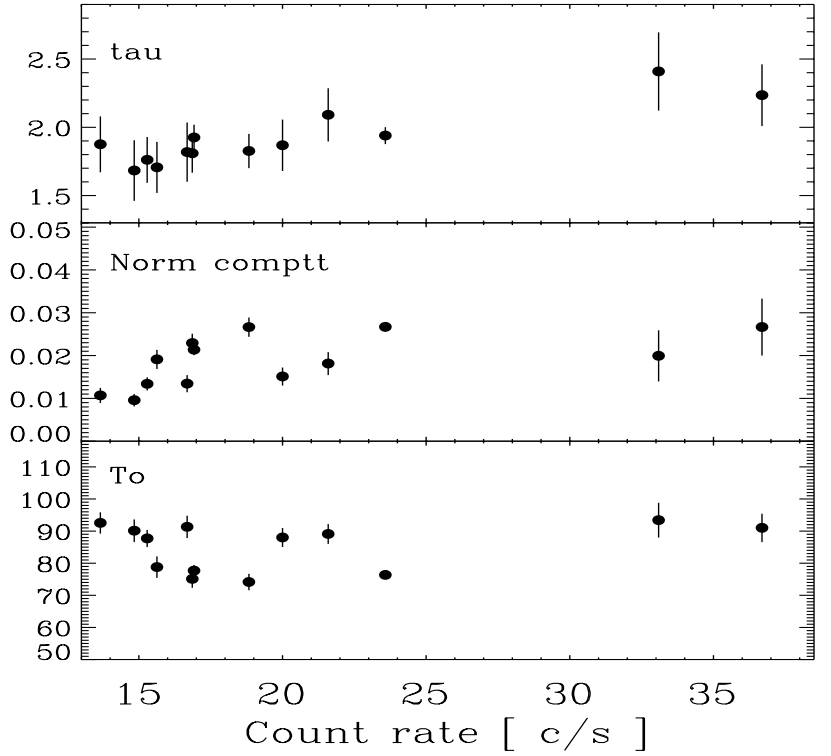

Fig. 7. Parameters of the compTT component of a compTT + power law fit as a function count rate.

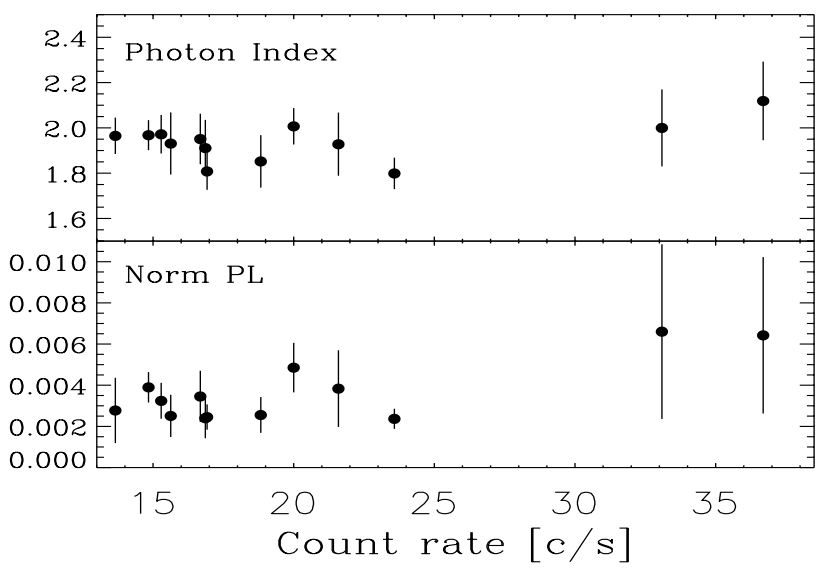

Fig. 8. Parameters of the power law component of a compTT + power law fit as a function count rate.

Comptonization models: one with low $(\sim 4.5 \mathrm{keV})$ temperature and moderate optical depths $(\tau \sim 2.0)$ and a second, high temperature component with $k T \gtrsim 50 \mathrm{keV}$ and low scattering depths $(\tau \lesssim 0.7)$. A determination of exact parameters of the scatterer is not possible: from the high $k T$ component only the low-energy power law can be seen in the XMM energy band and the measured slope is merely an indicator for a wide range of possible $(k T, \tau)$ combinations (Titarchuk \& Lyubarskij 1995). The low temperature component shows in principle measurable characteristic changes of the spectral slope inside the PN energy band; however, the superposition of the two components and the position of the spectral break at rather high energies $(\gtrsim 5 \mathrm{keV})$ with low photon statistics leaves considerable uncertainties in the parameter determination as well. Thus, this low-temperature component could be quite well replaced with a considerably hotter component with much smaller optical depth; the physical implications of this (less favored) combination will be discussed below.
Nevertheless, the above envisaged two components, low $k T$ and high $\tau$ plus high $k T$ and low $\tau$ represent an appealing picture for the X-ray emission of NLS1 galaxies. The high temperature component is very similar to that describing the emission of broad line Seyfert 1 (BLS1) galaxies. For those BeppoSax measurements (Petrucci et al. 2001) gave temperatures in excess of $150 \mathrm{keV}$ and optical depths around $\tau \sim 0.2$. As the PDS detector covers the high energy band where spectral curvatures occur, the errors on temperature and optical depth are very small; the largest uncertainties seem to be related to the exact spectral shapes predicted by the different Comptonization models.

Unfortunately, the low signal-to-noise high energy BeppoSax data for PKS 0558-504 do not further constrain the parameters of the hot Comptonization component. A fit to the combined MECS and PDS data with a compTT model resulted in a $k T=47 \mathrm{keV}, \tau=0.56$ and a $\chi_{\text {red }}^{2}=0.97$. However, the $90 \%$ confidence intervals for these values are $20.8 \mathrm{keV} \lesssim k T \lesssim$ $162 \mathrm{keV}$ and $0.01 \lesssim \tau \lesssim 2.85$.

The PKS 0558-504 fits at higher energies of either a power law or the hot Comptonizing component are in agreement with the results for the BLS1 fits, as can be seen from the conversion of the power law slopes to $(k T, \tau)$ (Titarchuk \& Lyubarskij 1995, Eqs. (17) and (22)). Thus, this component seems to be present in both object classes, in NLS1 and BLS1 galaxies.

In addition to this hard component NLS1 galaxies show a second, softer Comptonization component. This soft component contributes about twice the luminosity of the hard component over the $0.1-100 \mathrm{keV}$ energy range and clearly dominates the emission in the XMM band. As the Compton luminosity of a source is proportional $L \sim y \times U \times R^{2}$ (Haardt, private comm.), where $U$ is the radiation energy density of the soft photons, $R$ the size of the source and $y \sim k T \max \left(\tau, \tau^{2}\right)$ is the Compton $y$ parameter, the emission areas for the two components with the above given parameters must be of similar sizes.

In contrast to BLS1 galaxies no iron lines from a reflection component could be detected. It appears that the "cold reflector" in BLS1 galaxies, responsible for the iron line emission, is replaced by a "warm" ( $k T \sim 4.5 \mathrm{keV})$, scattering component. Further, the higher optical depths imply that any reflection features (like the iron line) tend to be more suppressed by Compton scattering in the corona itself (Matt et al. 1997).

\subsection{Temporal variability: Short time scales}

The source shows variability on various time scales. During the individual observations we find flux changes of typically $\$ 20 \%$ (the maximum is nearly $50 \%$ in orbit 33 ) on time scales of a few hours. A closer inspection shows that these flux variations are caused by changes of the soft compTT component only; the hard component remains practically constant.

This is nicely illustrated in Fig. 9 which shows, as example, the comparison of the two spectra for the low and high state of orbit 32 . We fitted the first, about $5 \mathrm{ks}$, of the data when the source was at a low flux level with two compTT models. We then froze the fit parameters and plot the data from the peak of the light curve and the deviations of the these data from the 


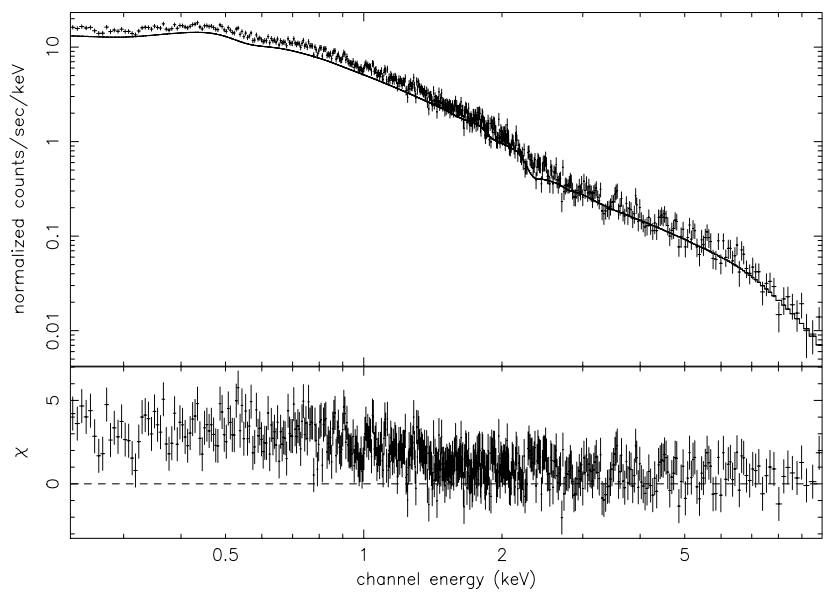

Fig. 9. The high state data of orbit 32 overlaid on a two component compTT model with parameters which were obtained from a fit to the low state data from the first $5 \mathrm{ks}$ of the observation. The lower panel shows the $\delta \chi^{2}$ per channel between data and this model.

model (in $\delta \chi^{2}$ ) in the bottom panel of Fig. 9. As can be seen, the intensity changes occur only in the soft part of the spectrum.

More details of the spectral behavior can be seen in Fig. 10 which shows the changes in the spectrum during orbit 42 . The spectra correspond first to the low count rate state at the beginning of the orbit, then to the rising phase, and finally to the high count rate state at the end of this observation. The spectra were fitted with a compTT plus a power law model, with fixed galactic absorption and a fixed temperature of $4.5 \mathrm{keV}$ for the Comptonizing electrons to avoid the degeneracy problem between optical depth and electron temperature. The model parameters of the power law component remain unchanged within the errors while in the Comptonization component the optical depth increases slightly with count rate from 2.1 to 2.5 . In these fits the increase in the optical depth is responsible for the change of the spectral shape: it dominates the spectrum up to higher energies as the count rate increases.

The plot further illustrates the difficulties of a hardness ratio analysis: at low count rates the soft and the hard band are each representing the contribution of the individual Compton components; at very high intensities most of the XMM spectral range is dominated by the soft component.

Thus the flux changes occurring on time scales of $z 2 \mathrm{~h}$ in a relatively smooth manner are caused by variations of the strength of the soft compTT component. Whether these variations are caused by changes of the temperature of the Comptonizing gas (at constant scattering depths $\tau$ and normalizations) or by changes of $\tau$ and the normalization at a constant gas temperature cannot be distinguished from the spectral fits. The first scenario would require a heating of the same gas volume, possibly by electromagnetic processes (i.e. Alfvén waves); the latter requires changes in the size of the emitting area and/or the volume of the gas responsible for the emission. This could be most easily achieved by an increase of the number of active emission regions on the disc (Haardt et al. 1994), which are formed at similar temperatures but with initially higher densities and then evolve gas dynamically. A popular class of models which follows these patterns are

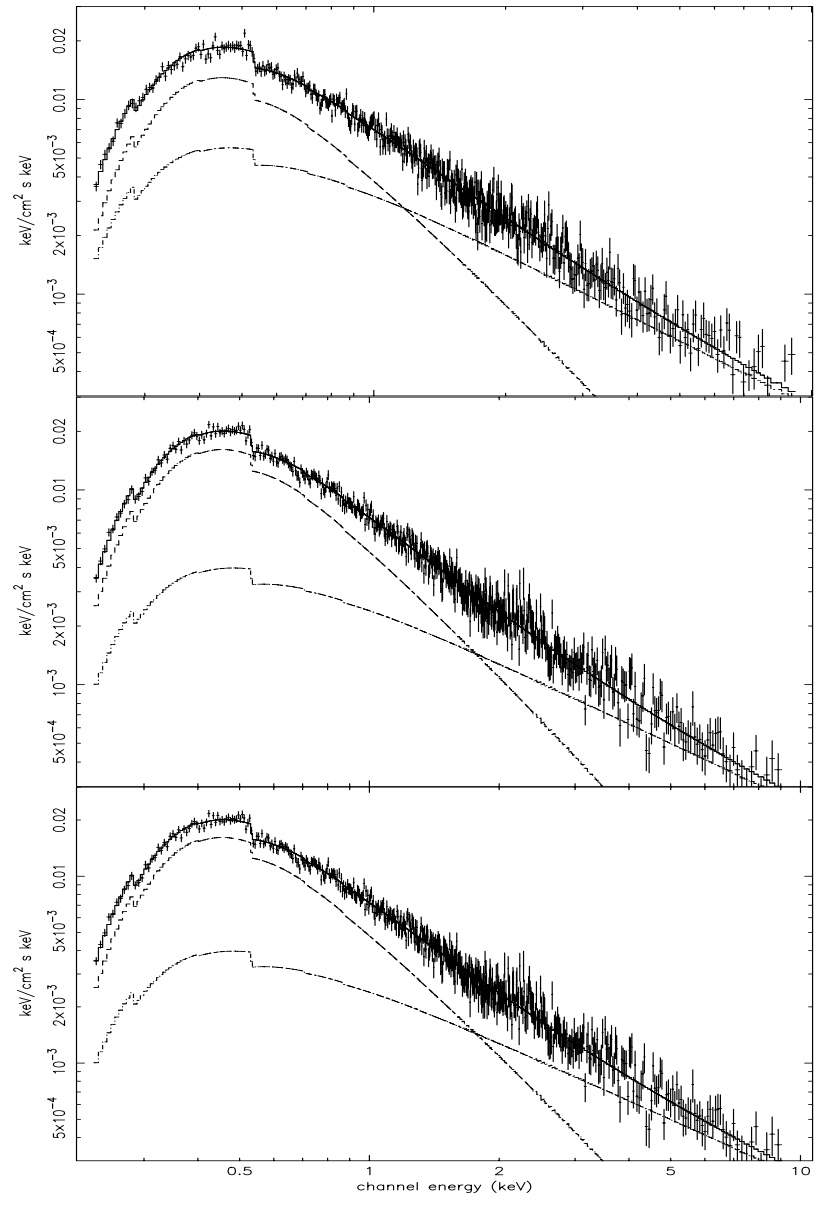

Fig. 10. Unfolded spectra from orbit 42 in the preflare (top), rise (middle) and flare (bottom) stages. The average count rates for the plots, from top to bottom, are 24.2, 25.0, 28.0 counts/s.

magnetically heated coronas where the energy is primarily stored in magnetic fields which rise buoyantly from the disc, reconnect and release their energy in flares (Merloni \& Fabian 2001). These heat the corona and can trigger an avalanche (Poutanen \& Fabian 1999) which create in turn a bigger active region.

The energy released in a typical burst $\Delta L \Delta \mathrm{t}$ is of the order of $10^{45} \mathrm{erg}$. Using the characteristic size of the system (see Sects. 4.3 and 4.4 ) of a few $\times 10^{13} \mathrm{~cm}$ it turns out that this energy can be stored easily in a magnetic field of the order of $\lesssim 10^{3}$ Gauss or, taking the physical parameters from the Comptonization fits, as thermal energy of the gas. However, recently Merloni \& Fabian (2001) strongly argued in favor of magnetic fields for storing the energy in the corona. The time scales for Compton cooling are rather short, of the order of $10^{2}$ s only (Wang et al. 2001), therefore the evolution of the bursts must be governed by the rate at which energy can be supplied to the emission region.

\subsection{Temporal variability: Longer time scales}

We find evidence for a time scale of $\sim 24 \mathrm{ks}$ in the data from the recurrent occurrence of stronger intensity peaks. Unfortunately, the statistical significance for the existence of such signal cannot be assessed reliably due to the extreme sparsity of the data. 
Although not strictly periodic - there are some orbits where no peak occurs at the expected time - this time scale seems to represent a quite stable clock in the system. It might either be a rather well defined time scale of a dominant instability occurring in the disc or it might be related to the orbital time scale of the emitting matter.

By adopting the empirical broad-line region size versus optical luminosity relation of Kaspi et al. (2000) and using the $\mathrm{H} \beta$ line width of $1250 \mathrm{~km} \mathrm{~s}^{-1}$ (Corbin 1997) a central black hole mass of $4.5 \times 10^{7} M_{\odot}$ was derived for PKS 0558-504 by Wang et al. (2001). Assuming Keplerian motion the above time scale of $\sim 24 \mathrm{ks}$ would correspond to the orbital period at $\sim 3.2$ Schwarzschild radii, i.e. just outside the last stable orbit. Considering the uncertainties in the above mass determination this distance strongly supports the interpretation that the $24 \mathrm{ks}$ time scale is related to the orbital motion of the emission region.

\subsection{Temporal variability: The longest time scales}

On even longer time scales, of the order of several satellite orbits, the source changes by more than a factor of two, but we never see these transitions to occur during an individual orbit. For example, during orbits 42 and 45 the source is obviously in a high state which might thus last for more than a week. During the second part of orbit 33, i.e. about 2.5 weeks earlier, the flux is more than 2.5 times smaller. That these long term intensity variations are a common phenomenon of NLS1 galaxies is demonstrated in the long light curves of Ark 564 (Gliozzi et al. 2002) or MCG-6-30-15 (Vaughan et al. 2003).

Substantial changes of the radiating area or the physical conditions of this area, respectively, are expected to happen on the dynamical time scale for Keplerian inflow, $\tau \sim 9 \times$ $10^{3}\left(r / R_{\mathrm{s}}\right)^{3 / 2}\left(M_{\mathrm{bh}} / 10^{7} M_{\odot}\right) \mathrm{s}$, where $R_{\mathrm{s}}$ is the Schwarzschild radius of the central black hole of mass $M_{\mathrm{bh}}$. With the above deduced parameters for PKS 0558-504 we find a time scale of $\sim 2.3 \times 10^{5} \mathrm{~s}$, which is consistent with the observational result that large changes of the source flux occur on time scales longer than an XMM orbit.

\section{Conclusions}

We have analyzed in some detail the observations of PKS 0558-504 performed over nine irregularly spaced XMM orbits spanning a time of more than two years.

The source showed strong variability of $\$ 20 \%$ in an individual observation with typical time scales of $\gtrsim 2 \mathrm{~h}$. The flux variations often exceed the efficiency limit for scattering limited spherical accretion. However, as the emission is dominated by Compton up-scattered soft photons in a hot corona heated by magnetic reconnection, and the energy is stored in the magnetic fields which do not contribute to the scattering opacity, the effective efficiency could be as large as $\eta \lesssim B^{2} /\left(8 \pi \rho c^{2}\right) \lesssim 1$, where $\rho$ is the mass density of the corona region (Wang et al. 2001).

The flaring pattern seems to repeat with a time scale of $\sim 24 \mathrm{ks}$, however not in a strictly periodic manner. Interpreting this time scale as the orbital time scale of Keplerian motion we find that the emission occurs at distances of $\gtrsim 3.2$ Schwarzschild radii from the central black hole. On longer time scales, beyond the temporal spacing of the XMM orbits, intensity changes by more than a factor of two are observed, which can be related to the dynamical time scale of Keplerian inflow in the disc. We do not see any large flare from the source as reported from Ginga (Remillard et al. 1991) and ASCA (Wang et al. 2001) observations. Thus that phenomenon must be a rare event.

The spectrum of PKS 0558-504 (like that of several other NLS1 galaxies as well) can be well fitted by the sum of two Comptonization models, one with high temperature $(k T \gtrsim$ $50 \mathrm{keV})$ and low optical depths $(\tau \sim 0.7)$, similar to that found for BLS1 galaxies, and an additional cooler component $(k T \sim 4.5 \mathrm{keV})$ and larger optical depths $(\tau \gtrsim 2)$. In contrast to BLS1 galaxies no iron lines from a reflection component could be detected. It appears that the "cold reflector" in BLS1 galaxies, responsible for the iron line emission, is replaced by a "warm" $(k T \sim 4.5 \mathrm{keV})$, scattering component. Flux variations are predominantly caused by changes of this latter component, whose presence is obviously the distinguishing criterion for the X-ray properties of NLS1 galaxies.

As the spectral capabilities of current instruments are insufficient to resolve the degeneracy in the spectral shapes of multiple Comptonization model fits, the second (cooler) component could equally well be fitted by another hot component with extremely small optical depths $(\tau \lesssim 0.06)$. This would, however, not change the general scenario of a magnetically very active corona on the accretion discs of NLS1 galaxies but require different physical parameters for the generation of the coronal active regions. Unfortunately, current corona models do not seem to have sufficient predictive power to allow to distinguish between these theoretical possibilities. For the low mass of the accreting black hole the correspondingly high accretion rate is expected to be responsible for higher magnetic field strengths in NLS1 galaxies (Mineshige et al. 2000) providing a mean for storing energy in the corona and leading to strong variability of the emission from the systems (Merloni \& Fabian 2001; Merloni 2003). If the turbulent magnetic pressure greatly exceeds that of the gas turbulent Comptonization might play an important role in producing the soft X-rays (Socrates et al. 2003). This largely unexplored process would provide a direct link between details of the disc physics and the observed spectrum.

An unanswered question is related to the radio-loudness of PKS 0558-504, and how that affects the emission characteristics. The spectral decomposition into two Comptonization components and the short-term intensity variations are found in other, radio-quiet NLS1 galaxies as well, therefore we regard this as being a typical NLS1 characteristic and not related to a possible radio jet, although outflows are expected from powerful magnetically dominated coronas (Merloni \& Fabian 2002). However, the additional emission from a jet might be responsible for the large flare events, detected by Ginga (Remillard et al. 1991) and ASCA (Wang et al. 2001). It should be noted that during the flare the spectrum was significantly harder than 
in the "quiescence state" (Wang et al. 2001), thus indicating an additional, different emission mechanism.

Unfortunately, the X-ray spectra of NLS1 galaxies are nearly featureless and thus they bear only little discriminating power to allow parameter studies for the various corona models. The best way to get access to the physical processes responsible for the X-ray emission might be, like in BL Lac objects, to pursue detailed temporal analyses of the emission.

Acknowledgements. This work is based on observations with XMM-Newton, an ESA science mission with instruments and contributions directly funded by ESA Member States and the USA (NASA). We thank Graziella Branduardi-Raymont for her help for producing RGS light curves and the referee, P. Uttley, for constructive comments which improved the quality of the paper. P.A. \& E.F. acknowledge support by the International Max-Planck Research School on Astrophysics (IMPRS).

\section{References}

Boller, Th., Brandt, W. N., \& Fink, H. 1996, A\&A, 305, 53

Boller, Th., Brandt, W. N., Leighly, K. M., \& Ward, M. J. 2000, Proceedings of the Workshop on Observational and Theoretical Progress in the Study of NLS1 Galaxies, New Astron. Rev., 44, 381

Brandt, W. N., \& Boller, Th. 1998, Astron. Nachr., 319, 7

Brandt, W. N., Boller, Th., Fabian, A. C., \& Ruszwoski, M. 1999, MNRAS, 303, L53

Brinkmann, W., Yuan, W., \& Siebert, J. 1997, A\&A, 319, 413

Brinkmann, W., Grupe, D., Branduardi-Raymont, G., \& Ferrero, E. 2003, A\&A, 398, 81

Corbin, M. R. 1997, ApJS, 113, 245

den Herder, J. W., Brinkman, A. C., Kahn, S. M., et al. 2001, A\&A, 365, L7

Ehle, M., Breitfellner, M., Dahlem, M., et al. 2001, XMM-Newton Users' Handbook, http://xmm.vilspa.esa.es/ xmm_user_support/external/documentation/ uhb_frame.shtml

Fabian, A. C. 1979, Proc. R. Soc. London, Ser. A, 366, 449

Ghizzardi, S., \& Molendi, S. 2001, Proc. Conf., New Visions of the $\mathrm{X}$-ray Universe, ESTEC

Gliozzi, M., Boller, Th., Brinkmann, W., \& Brandt, W. N. 2000, A\&A, 356, L17

Gliozzi, M., Brinkmann, W., O’Brien, P. T., et al. 2001, A\&A, 365, L128

Gliozzi, M., Brinkmann, W., Räth, C., et al. 2002, A\&A, 391, 875
Goodrich, R. W. 1989, ApJ, 342, 224

Grupe, D., Beuermann, K., Mannhein, K., \& Thomas, H.-C. 1999, A\&A, 350, 805

Haardt, F., \& Maraschi, L. 1993, ApJ, 413, 507

Haardt, F., Maraschi, L., \& Ghisellini, G. 1994, ApJ, 432, L95

Kaspi, S., Smith, P. S., Netzer, H., et al. 2000, ApJ, 533, 631

Kazanas, D., Hua, X.-M., \& Titarchuk, L. 1997, ApJ, 480, 735

Matt, G., Fabian, A. C., \& Reynolds, C. S. 1997, MNRAS, 289, 175

Merloni, A., \& Fabian, A. C. 2001, MNRAS, 321, 549

Merloni, A., \& Fabian, A. C. 2002, MNRAS, 332, 165

Merloni, A. 2003, MNRAS, in press [astro-ph/0302074]

Mineshige, S., Takeuchi, M., \& Nishimori, H. 1994, ApJ, 435, 125

Mineshige, S., Kawagichi, T., Takeuchi, M., \& Hayashida, K. 2000, PASJ, 52, 499

Moran, E. C., Halpern, J. P., \& Helfand, D. J. 1996, ApJS, 106, 341

O’Brien, P. T., Reeves, J. N., Turner, M. J. L., et al. 2001, A\&A, 365, L122

Osterbrock, D. E., \& Pogge, R. W. 1985, ApJ, 297, 166

Page, K. L., Pounds, K. A., Reeves, J. N., \& O’Brien, P. T. 2002, MNRAS, 330, L1

Page, K. L., O’Brien, P. T., Reeves, J. N., \& Breeveld, A. A. 2003, MNRAS, 340, 1052

Persson, S. E. 1988, ApJ, 330, 751

Petrucci, P. O., Haardt, F., Maraschi, L., et al. 2001, ApJ, 556, 716

Pounds, K. A., Done, C., \& Osborne, J. 1995, MNRAS, 277, L5

Poutanen, J., \& Fabian, A. C. 1999, MNRAS, 306, L31

Remillard, R. A., Bradt, H. V., Buckley, D. A. H., et al. 1986, ApJ, 301,742

Remillard, R. A., Grossan, B., Bradt, H. V., et al. 1991, Nature, 350, 589

Siebert, J., Leighly, K. M., Laurent-Muehleisen, S. A., Brinkmann, W., Boller, Th., \& Matsuoka, M. 1999, A\&A, 348, 678

Simonetti, J. H., Cordes, M. J., \& Heeschen, D. S. 1985, ApJ, 296, 46

Socrates, A., Davis, S. W., \& Blaes, O. 2003 [astro-ph/0307158]

Strüder, L., Briel, U. G., Dennerl, K., et al. 2001, A\&A, 365, L18

Titarchuk, L. 1994, ApJ, 434, 313

Titarchuk, L., \& Lyubarskij, Y. 1995, ApJ, 450, 876

Ulvestad, J. S., Antonucci, R. R. J., \& Goodrich, R. W. 1995, AJ, 109, 81

Uttley, P., McHardy, I. M., \& Papadakis, I. E. 2002, MNRAS, 332, 231

Vaughan, S., Reeves, J., Warwick, R., \& Edelson, R. 1999, MNRAS, 309, 113

Vaughan, S., Fabian, A. C., \& Nandra, K. 2003, MNRAS, 339, 1273

Wang, T., Matsuoka, M., Kubo, H., Mihara, T., \& Negoro, H. 2001, ApJ, 554, 233 